\title{
FROM TWO- TO FOUR-VALUED LOGIC
}

\author{
CHRIS BRINK \\ Department of Mathematics, University of Cape Town \\ Rondebosch 7700, Cape Town, South Africa \\ E-mail: CBRINK@UCTHPX.UCT.AC.ZA
}

\begin{abstract}
The purpose of this note is to show that a known and natural four-valued logic co-exists with classical two-valued logic in the familiar context of truth tables. The tool required is the power construction.
\end{abstract}

The context of this paper is that of 2 -valued truth tables in $n$ propositional variables, say $p_{1}, p_{2}, \ldots, p_{n}$. The two classical truth values are 0 and 1 . The truth value assignments (or valuations) are the $2^{n}\{0,1\}$-vectors of length $n$. Propositional formulae are freely generated from the propositional variables by application of the usual classical connectives $\sim$ (not), \& (and) and $\vee$ (or), each of which is defined by a given truth table. Accordingly, each propositional formula has a truth table. Formulae with the same truth table are logically equivalent, and we do not distinguish between them.

The valuations form a Boolean algebra Val $=\left(\mathbf{2}^{n}, \wedge, \vee,^{\prime}, \mathbf{0}, \mathbf{1}\right)$, where the Boolean operations $\wedge, \vee$ and ' are defined componentwise from their counterparts in the 2-element Boolean algebra $\mathbf{2}=\{0,1\}$, and $\mathbf{0}$ and $\mathbf{1}$ are respectively the vectors $(0,0, \ldots, 0)$ and $(1,1, \ldots, 1)$ of length $n$. The elements of $\mathbf{2}^{n}$ are denoted by $\boldsymbol{x}, \boldsymbol{y}, \boldsymbol{z}, \ldots$ Up to equivalence, formulae may be regarded as truth functions from $\mathbf{2}^{n}$ to $\mathbf{2}$, and the connectives are characterised by operations in the image algebra $\mathbf{2}$. That is, if $\operatorname{bool}(\mathcal{A},-): \mathbf{2}^{n} \rightarrow \mathbf{2}$ is the truth function corresponding to a propositional formula $\mathcal{A}$, then

$$
\begin{aligned}
\operatorname{bool}(\sim \mathcal{A}, \boldsymbol{x}) & =\operatorname{bool}(\mathcal{A}, \boldsymbol{x})^{\prime}, \\
\operatorname{bool}(\mathcal{A} \& \mathcal{B}, \boldsymbol{x}) & =\operatorname{bool}(\mathcal{A}, \boldsymbol{x}) \wedge \operatorname{bool}(\mathcal{B}, \boldsymbol{x}), \\
\operatorname{bool}(\mathcal{A} \vee \mathcal{B}, \boldsymbol{x}) & =\operatorname{bool}(\mathcal{A}, \boldsymbol{x}) \vee \operatorname{bool}(\mathcal{B}, \boldsymbol{x}) .
\end{aligned}
$$

1991 Mathematics Subject Classification: 03B46, 03B05.

The paper is in final form and no version of it will be published elsewhere. 
I now proceed to define some new connectives by using operations on the domain algebra Val.

It will be convenient to think of a formula (up to equivalence) as a subset of $\mathbf{2}^{n}$. This is an easy variation on thinking of formulae as truth functions, since truth functions may be thought of as characteristic functions. Thus we may define, for any formula $\mathcal{A}$,

$$
m(\mathcal{A})=\left\{\boldsymbol{x} \in \mathbf{2}^{n} \mid \operatorname{bool}(\mathcal{A}, \boldsymbol{x})=1\right\} .
$$

This allows us to think of the classical connectives as the classical set-theoretic operations, since

$$
\begin{aligned}
m(\sim \mathcal{A}) & =m(\mathcal{A})^{\prime}, \\
m(\mathcal{A} \& \mathcal{B}) & =m(\mathcal{A}) \cap m(\mathcal{B}), \\
m(\mathcal{A} \vee \mathcal{B}) & =m(\mathcal{A}) \cup m(\mathcal{B}) .
\end{aligned}
$$

But these operations on subsets of $\mathbf{2}^{n}$ (and thus, by implication, the classical connectives) take no account of the algebraic structure of $\mathbf{2}^{n}$. We will use some operations which do.

For the desired results to follow it is required to make one small but crucial modification to $\mathbf{2}^{n}$. This can be done quite generally, as follows.

Let $\mathcal{B}=\left(B, \vee, \wedge,{ }^{\prime}, 0,1\right)$ be any Boolean algebra. Over $B$ define partial operations $\wedge_{\mathrm{p}}$ and $\vee_{\mathrm{p}}$ as follows:

$$
\begin{aligned}
& a \wedge_{\mathrm{p}} b= \begin{cases}a \wedge b & \text { if } a \wedge b \neq 0, \\
0 & \text { if } a=0 \text { or } b=0, \\
\text { undefined } & \text { otherwise. }\end{cases} \\
& a \vee_{\mathrm{p}} b= \begin{cases}a \vee b & \text { if } a \vee b \neq 1, \\
1 & \text { if } a=1 \text { or } b=1, \\
\text { undefined } & \text { otherwise. }\end{cases}
\end{aligned}
$$

The intention here is best explained in set-theoretic terms. Think of $\wedge$ as intersection of sets. Convention has it that if two nonempty sets $A$ and $B$ do not intersect then their intersection $A \cap B$ is defined, and equal to the empty set $\emptyset$. The variation adopted here is that if two nonempty sets $A$ and $B$ do not intersect then they don't have an intersection: $A \cap B$ is undefined. Dually for $\vee$, via $\cup$. We will call $\mathcal{B}_{\mathrm{p}}=\left(B, \wedge_{\mathrm{p}}, \vee_{\mathrm{p}},{ }^{\prime}, 0,1\right)$ a partial Boolean algebra, with $\wedge_{\mathrm{p}}$ and $\vee_{\mathrm{p}}$ called partial meet and partial join.

Specifically, let $\wedge_{\mathrm{p}}$ and $\vee_{\mathrm{p}}$ be partial meet and partial join in $\mathbf{2}^{n}$.

Now consider any formulae $\mathcal{A}$ and $\mathcal{B}$, and picture their truth tables in the usual way, with truth value assignments (elements of $\mathbf{2}^{n}$ ) in a column on the left, and column vectors of 0 's and 1's representing $\mathcal{A}$ and $\mathcal{B}$ respectively. Then define new formulae (i.e., truth tables of) $\neg \mathcal{A}, \mathcal{A} \odot \mathcal{B}$ and $\mathcal{A} \oplus \mathcal{B}$ as follows:

(6) Given the column representing $\mathcal{A}$, to construct the column representing $\neg \mathcal{A}$ proceed down the rows as follows: 
(a) find the first remaining row in which $\mathcal{A}$ has value 1 , and say this appears against truth value assignment $\boldsymbol{x}$;

(b) put a 1 in the row corresponding to $\boldsymbol{x}^{\prime}$;

(c) repeat (a) and (b) until this process terminates, then fill up the remaining places in the column with 0's.

(7) Given the columns representing $\mathcal{A}$ and $\mathcal{B}$, to construct the column representing $\mathcal{A} \odot \mathcal{B}$ proceed down the rows as follows:

(a) find the first remaining row in which $\mathcal{A}$ has value 1 , and say this appears against truth value assignment $\boldsymbol{x}$;

(b) (i) find the first remaining row in which $\mathcal{B}$ has value 1 , and say this appears against truth value assignment $\boldsymbol{y}$;

(ii) put a 1 in the row corresponding to $\boldsymbol{x} \wedge_{\mathrm{p}} \boldsymbol{y}$;

(iii) repeat (i) and (ii) until the process terminates;

(c) repeat (a) and (b) until this process terminates, then fill up the remaining places in the columns with 0 's.

(8) Given the columns representing $\mathcal{A}$ and $\mathcal{B}$, to construct the column representing $\mathcal{A} \oplus \mathcal{B}$ proceed as in (7), only using $\vee_{\mathrm{p}}$ instead of $\wedge_{\mathrm{p}}$.

Here are some examples in the 3 -valued case.

\begin{tabular}{ccccccccccccccc}
\hline$p_{1}$ & $p_{2}$ & $p_{3}$ & $\mathcal{A}$ & $\mathcal{B}$ & $\mathcal{C}$ & $\neg \mathcal{A}$ & $\neg \mathcal{B}$ & $\neg \mathcal{C}$ & $\mathcal{A} \odot \mathcal{B}$ & $\mathcal{A} \odot \mathcal{C}$ & $\mathcal{B} \odot \mathcal{C}$ & $\mathcal{A} \oplus \mathcal{B}$ & $\mathcal{A} \oplus \mathcal{C}$ & $\mathcal{B} \oplus \mathcal{C}$ \\
\hline 1 & 1 & 1 & 0 & 1 & 0 & 1 & 0 & 0 & 0 & 0 & 0 & 1 & 0 & 1 \\
1 & 1 & 0 & 0 & 0 & 1 & 1 & 0 & 0 & 0 & 0 & 1 & 0 & 1 & 1 \\
1 & 0 & 1 & 1 & 1 & 0 & 0 & 0 & 1 & 1 & 0 & 0 & 1 & 0 & 0 \\
1 & 0 & 0 & 0 & 1 & 0 & 1 & 0 & 0 & 1 & 1 & 1 & 1 & 0 & 0 \\
0 & 1 & 1 & 1 & 0 & 0 & 0 & 1 & 0 & 1 & 0 & 0 & 0 & 1 & 0 \\
0 & 1 & 0 & 0 & 0 & 1 & 1 & 1 & 0 & 0 & 1 & 1 & 0 & 1 & 0 \\
0 & 0 & 1 & 1 & 0 & 0 & 0 & 0 & 1 & 1 & 0 & 0 & 0 & 0 & 0 \\
0 & 0 & 0 & 1 & 0 & 0 & 0 & 1 & 0 & 1 & 1 & 0 & 0 & 0 & 0 \\
\hline
\end{tabular}

Actually, it is much cleaner to present these operations via set-theoretic representations, capitalising on the algebraic structure of $\mathbf{2}^{n}$. Namely, we take the power operations of complementation ', partial meet $\wedge_{p}$ and partial join $\vee_{p}$ over $\mathbf{2}^{n}$. This notion is one of the power constructions expounded in Brink [1992].

In general, given any $m$-ary operation $f: X^{m} \rightarrow X$, its power operation $f^{+}: \mathcal{P}(X)^{m} \rightarrow X$ is defined by

$$
\begin{aligned}
& f^{+}\left(A_{1}, \ldots, A_{m}\right)=\left\{f\left(a_{1}, \ldots, a_{m}\right) \mid a_{i} \in A_{i}, 1 \leq i \leq m\right\} \\
& \text { for every } A_{i} \subseteq X, 1 \leq i \leq m .
\end{aligned}
$$

In case $f$ is a partial operation it may happen that the definiens is the empty set.

In $\mathbf{2}^{n}$ adopt the notation that $\neg X=\left\{\boldsymbol{x}^{\prime} \mid \boldsymbol{x} \in X\right\}$ for any $X \subseteq \mathbf{2}^{n}$. Now consider any formulae $\mathcal{A}$ and $\mathcal{B}$, and define via their meanings (i.e. up to equivalence) new formulae $\neg \mathcal{A}, \mathcal{A} \odot \mathcal{B}$ and $\mathcal{A} \oplus \mathcal{B}$ by 


$$
\begin{aligned}
m(\neg \mathcal{A}) & =\neg m(\mathcal{A}), \\
m(\mathcal{A} \odot \mathcal{B}) & =m(\mathcal{A}) \wedge_{\mathrm{p}}^{+} m(\mathcal{B}), \\
m(\mathcal{A} \oplus \mathcal{B}) & =m(\mathcal{A}) \vee_{\mathrm{p}}^{+} m(\mathcal{B}) .
\end{aligned}
$$

Compare this with (3); then we see that the new connectives are defined via the representative subsets of $\mathbf{2}^{n}$ by utilising their algebraic structure, whereas the classical connectives are simply defined set-theoretically.

For obvious historical reasons we may call $\sim$, \& and $\vee$ Boolean connectives, and for any formula $\mathcal{A}$ think of $\operatorname{bool}(\mathcal{A}, \boldsymbol{x})$ as the Boolean truth value of $\mathcal{A}$ at $\boldsymbol{x}$. For reasons which will soon be clear let us call $\neg, \odot$ and $\oplus$ Belnap connectives, and define for any formula $\mathcal{A}$ its Belnap truth value as follows:

$$
\operatorname{bel}(\mathcal{A})= \begin{cases}\boldsymbol{T} & \text { if } \mathbf{1} \in m(\mathcal{A}) \text { and } \mathbf{0} \notin m(\mathcal{A}), \\ \boldsymbol{B} & \text { if } \mathbf{1} \in m(\mathcal{A}) \text { and } \mathbf{0} \in m(\mathcal{A}), \\ \boldsymbol{N} & \text { if } \mathbf{1} \notin m(\mathcal{A}) \text { and } \mathbf{0} \notin m(\mathcal{A}), \\ \boldsymbol{F} & \text { if } \mathbf{1} \notin m(\mathcal{A}) \text { and } \mathbf{0} \in m(\mathcal{A})\end{cases}
$$

Note that whereas the Boolean truth value of a formula $\mathcal{A}$ is relative to some assignment $\boldsymbol{x} \in \mathbf{2}^{n}$, the Belnap truth values are not. Note further that if $\mathcal{A}$ is a contradiction, both $m(\mathcal{A} \odot \mathcal{B})$ and $m(\mathcal{A} \oplus \mathcal{B})$ are empty; this fits with (7) and (8). We now have the following Theorem (proved by cases):

(13) THEOREM. In the set of non-contradictory formulae the Belnap truth values of $\neg \mathcal{A}, \mathcal{A} \odot \mathcal{B}$ and $\mathcal{A} \oplus \mathcal{B}$ are as given in the Belnap truth tables, namely:

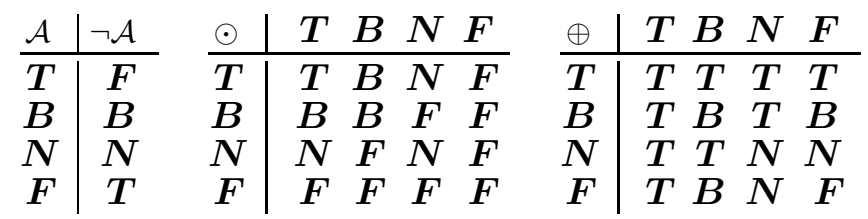

Now the reasons for the terminology are clear: these are the tables for the four-valued relevance logic of Anderson and Belnap [1975], subsequently treated in its own right by Belnap [1976, 1977].

But there's more to be said: what of implication?

In classical two-valued truth-table logic "implication" is thought of in two ways: as a connective, with $\mathcal{A} \Rightarrow \mathcal{B}$ either primitive or defined (say by $\sim \mathcal{A} \vee \mathcal{B}$ ); or as a relation, with " $\mathcal{A}$ implies $\mathcal{B}$ " defined as " $\mathcal{A} \Rightarrow \mathcal{B}$ is a tautology". Following up on (3) it is the latter case which is of interest here. Since

$$
\mathcal{A} \text { implies } \mathcal{B} \text { iff } m(\mathcal{A}) \subseteq m(\mathcal{B}),
$$

we may note that, like the classical connectives, this relationship does not exploit the algebraic structure of $\mathbf{2}^{n}$, and we introduce a relation which does. Namely, having used the power operations of the operations on $\mathbf{2}^{n}$, we now use the power relation of the natural Boolean ordering relation $\leq$ in $\mathbf{2}^{n}$. (The notion of power relation is as defined in Brink [1992].) So, for any formulae $\mathcal{A}$ and $\mathcal{B}$ we define 


$$
\begin{aligned}
m(\mathcal{A}) \Leftarrow m(\mathcal{B}) \quad \text { iff } \quad & (\forall \boldsymbol{x} \in m(\mathcal{A}))(\exists \boldsymbol{y} \in m(\mathcal{B}))[\boldsymbol{x} \leq \boldsymbol{y}] \text { and } \\
& (\forall \boldsymbol{y} \in m(\mathcal{B}))(\exists \boldsymbol{x} \in m(\mathcal{A}))[\boldsymbol{x} \leq \boldsymbol{y}] .
\end{aligned}
$$

Since we equate formulae with their set-theoretic representations we may also write $\mathcal{A} \Leftarrow \mathcal{B}$ iff $m(\mathcal{A}) \Leftarrow m(\mathcal{B})$. For an example, consider the 16 propositional formulae in 2 variables (say $p$ and $q$ ). Then $\mathbf{2}^{n}$ consists of the four vectors $(1,1)$, $(1,0),(0,1),(0,0)$, or equivalently the sets $\{p, q\},\{p\},\{q\}, \emptyset$. The formulae are then represented by sets of these sets, so that (e.g.) " $p \Rightarrow q$ " corresponds to $\{\{p, q\},\{q\}, \emptyset\}, " \sim p \& \sim q$ " corresponds to $\{\{p\},\{q\}, \emptyset\}$, and so on. (Note: this set representation arises from the column vector in the truth table, viewed as a characteristic function over $\mathcal{P}(\{p, q\})$.) With this translation it is easy to check that the 16 formulae in 2 variables are ordered by $\Leftarrow$ as in Figure 1 (where "Taut" is the tautology in two variables, and "Contrad" is the contradiction). Note that, because of the existential quantifiers in (15), Contrad is only related to itself.

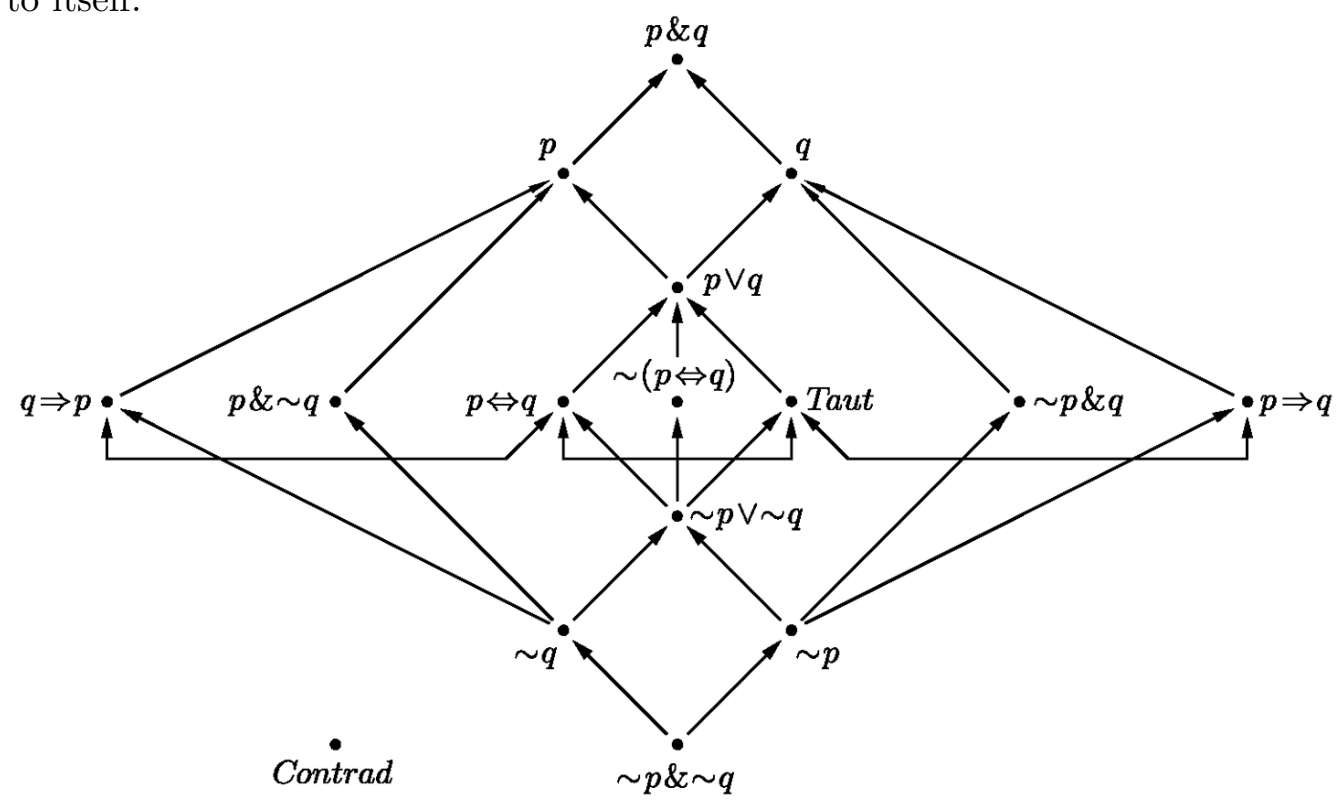

Fig. 1

We are now working with the power structure of $\mathbf{2}^{n}$ : the set of $n$-vectors endowed with the power operations of complementation and (partial) meet and join, and the power ordering $\Leftarrow$ of the Boolean ordering $\leq$. In $2^{n}$ the ordering $\leq$ and the operations $\wedge$ and $\vee$ are interdefinable, the latter being exactly least upper bounds and greatest lower bounds of the former. In the power structure this interdefinability is lost: $\Leftarrow$ is not a lattice order arising from $\odot$ and $\oplus$. (Indeed, $\Leftarrow$ is not even a partial order, nor do $\odot$ and $\oplus$ impose a lattice structure on $\mathbf{2}^{n}$.) However, the ordering $\Leftarrow$ does relate neatly to the four Belnap truth values. 
Evidently, (12) partitions the set of all propositional formulae into four (equivalence) classes, which by abuse of notation we may denote by $\boldsymbol{T}, \boldsymbol{B}, \boldsymbol{N}$ and $\boldsymbol{F}$, respectively. It is then easily checked that

(16) The Belnap truth values, viewed as classes on non-contradictory formulae, are ordered by $\leftarrow^{+}$, the power order of $\Leftarrow$, as in Figure 2 .

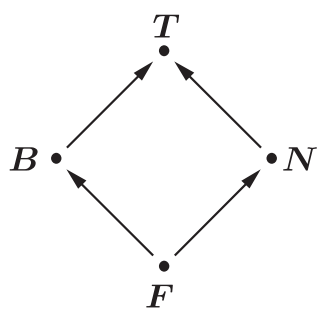

Fig. 2

But this is precisely the order imposed on the non-contradictory formulae by (13): the Belnap truth tables show that $\odot$ and $\oplus$ are lattice operations, and the lattice order thereby defined on $\{\boldsymbol{T}, \boldsymbol{B}, \boldsymbol{N}, \boldsymbol{F}\}$ coincides with that of $\Longleftarrow++$ in Figure 2.

I conclude that the power structure on truth value assignments in classical two-valued logic leads to the truth values of Belnap logic.

[Note: The actual development of the Belnap logic, in the context of classical propositional formulae, would have to address a problem sidestepped here: dealing with contradictions. The alert reader will have noticed in (13) that the set of noncontradictory formulae is not closed under $\odot$ : two non-contradictory formulae of Belnap value $\boldsymbol{N}$ may well yield a contradiction. The problem is real, but it is not surprising: the role of contradiction in inference is at the heart of relevance logic.]

For the foregoing to be more than just a bag of tricks it is necessary that some plausible interpretations attach to the technicalities. Here is a proposal.

Let there be given $n$ atomic sentences: $P_{1}, P_{2}, \ldots, P_{n}$, and think of these as stating atomic facts. That is, in what we will call the real world each of $P_{1}, \ldots, P_{n}$ is true. More: we think of the real world as being entirely constituted by the given atomic facts. (The philosophically minded may recall dictum 1.11 of Wittgenstein's Tractatus Logico-Philosophicus: "The world is determined by the facts, and by their being all the facts".) A possible world is one in which some atomic claims may not be true. The set of all possible worlds, which we may call the universe, is thus modelled by the set $\mathbf{2}^{n}$ of truth value assignments over the propositional variables $p_{1}, p_{2}, \ldots, p_{n}$. The real world is represented by $\mathbf{1}$, and we think of $\mathbf{0}$ as representing the anti-world (where every atomic truth of the real world is a falsehood). The idea that a sentence (generated from some atomic sentences by application of connectives) is either true or false in any possible world is modelled by the mapping bool $(-,-)$. For any sentence $A$ having the propositional form $\mathcal{A}$ (simply obtained by replacing atomic sentences by variables), and for any possible world $\boldsymbol{x} \in \mathbf{2}^{n}, \operatorname{bool}(\mathcal{A}, \boldsymbol{x})$ has value 1 or 0 , indicating that $A$ is true or false at $\boldsymbol{x}$. Thus classical truth values are relative to a possible world. 
By contrast, the Belnap truth value of a sentence $A$ is fixed over $\mathbf{2}^{n}$, it depends only on the truth or falsehood of $A$ in the real world and the anti-world. (Note again that $A$ can be true in either, both or neither.) As for the Belnap connectives, what is presented above is a Kripke-type semantics, in which the Boolean truth value of a (Belnap) compound sentence at a possible world is determined by the truth values of its constituents at some other worlds. Thus:

$$
\begin{array}{rrl}
\operatorname{bool}(\neg \mathcal{A}, \boldsymbol{x})=1 \quad \text { iff } & \operatorname{bool}\left(\mathcal{A}, \boldsymbol{x}^{\prime}\right)=1 . \\
\operatorname{bool}(\mathcal{A} \odot \mathcal{B}, \boldsymbol{x})=1 \quad \text { iff } \quad & \exists \text { worlds } \boldsymbol{y} \text { and } \boldsymbol{z} \text { such that } \operatorname{bool}(\mathcal{A}, \boldsymbol{y})=1 \text { and } \\
& \operatorname{bool}(\mathcal{B}, \boldsymbol{z})=1 \text { and } \boldsymbol{x}=\boldsymbol{y} \wedge_{\mathrm{p}} \boldsymbol{z} . \\
\operatorname{bool}(\mathcal{A} \oplus \mathcal{B}, \boldsymbol{x})=1 \quad \text { iff } \quad \exists \text { worlds } \boldsymbol{y} \text { and } \boldsymbol{z} \operatorname{such} \text { that } \operatorname{bool}(\mathcal{A}, \boldsymbol{y})=1 \text { and } \\
& \operatorname{bool}(\mathcal{B}, \boldsymbol{z})=1 \text { and } \boldsymbol{x}=\boldsymbol{y} \vee_{\mathrm{p}} \boldsymbol{z} .
\end{array}
$$

What distinguishes this from the Kripke semantics of (say) modal logic is that the set of possible worlds is endowed with algebraic structure, not (just) relational structure. As is common in the context of Kripke semantics we may think of the mapping $m$ : Sentences $\rightarrow \mathcal{P}\left(\mathbf{2}^{n}\right)$ as one of meaning: the meaning $m(A)$ of a sentence $A$ is the set of all possible worlds in which $A$ is true. But the set of possible worlds has algebraic structure, and the power construction enables us to lift some of that structure to sets of possible worlds, i.e. to meanings. The lifted operations give precisely the Belnap connectives and the ordering $\Leftarrow$, which thus inherit their properties from the structure of the universe.

As for $\Leftarrow$, Brink and Heidema [1987] have presented it as an ordering of verisimilitude (or truthlikeness). Think of the real world as being entirely constituted by two atomic facts: say "It is hot" and "It is windy". There are then 16 possible propositional theories about the weather, and these appear ordered by $\Leftarrow$ in Figure 1. Evidently the best theory is the one which says it is hot and windy, and the worst theory is the one which says it is cold and still. As for the rest, Brink and Heidema [1987] exhibit some properties of $\Leftarrow$ as "principles of verisimilitude"; if these are acceptable then Figure 1 presents an ordering of theories getting "closer to the truth".

In conclusion it is worth addressing briefly the matter of motivation. The Belnap logic originally arose from the quest for a notion of relevance between antecedent and consequent in an entailment; in Anderson and Belnap [1975] this is presented in terms of variable-sharing. The four-valued matrices are a later development, attributed to T. J. Smiley. These also fit into the context of so-called quasi-Boolean algebra, as expounded independently in Rasiowa [1974]. In his papers [1976, 1977] Belnap proposed and developed another motivation: that used in the area of knowledge representation by Levesque [1984] and Patel-Schneider [1989, 199?]. Other developments appear in Meyer and Martin [1986], Fitting [1989], Fox [1990] and Sylvan [199?].

A standard line of thought in many of these developments (due originally to Belnap and Dunn [1981]) is to equate the Belnap values with subsets of $\{0,1\}$, 
thus: $\boldsymbol{T}=\{1\}, \boldsymbol{B}=\{0,1\}, \boldsymbol{N}=\emptyset$ and $\boldsymbol{F}=\{0\}$. Then $\boldsymbol{T}$ means something like "always told true", $\boldsymbol{F}$ means "always told false", $\boldsymbol{B}$ means "told both true and false" and $\boldsymbol{N}$ means "told nothing". On this approach the Belnap values are thus seen as arising by putting classical values together. By contrast, the foregoing implicitly proposes to view the Belnap values as arising by taking classical values apart. Classically, to be true means to be true in the real world. There are then two subcases: a sentence $A$ true in the real world may still be either true or false in the anti-world. Thus the classical value 1 is composed of the Belnap values $\boldsymbol{T}$ and $\boldsymbol{B}$, and likewise the classical value 0 is composed of the Belnap values $\boldsymbol{N}$ and $\boldsymbol{F}$.

In thus bringing to bear on the notions of truth and falsehood not just the circumstances of the real world but also those of the anti-world, we treat of the two extremes of truthlikeness. Variations in between are ordered by $\Leftarrow$. So at least one virtue that may be claimed for the present approach is that it brings together, by application of the power construction, two hitherto separate notions: that of somehow treating truth and falsehood together, and that of increase and decrease in truthlikeness.

\section{References}

A. R. Anderson and N. D. Belnap, Jr. [1975], Entailment: The Logic of Relevance and Necessity, Vol. 1, Princeton Univ. Press.

N. D. Belnap [1976], How a computer should think, in: Contemporary Aspects of Philosophy, G. Ryle (ed.), Oriel Press, 30-56.

- [1977], A useful four-valued logic, in: Modern Uses of Multiple-valued Logic, J. M. Dunn and G. Epstein (eds.), Reidel, Dordrecht, 8-37.

N. D. Beln ap and J. M. Dunn [1981], Entailment and the disjunctive syllogism, in: Contemporary Philosophy: A New Survey, Vol. 1, G. Floistad and G. H. von Wright (eds.), Martinus Nijhoff, The Hague, 337-366.

C. Brink [1992], Power structures, to appear in Algebra Universalis.

C. Brink and J. Heidema [1987], A verisimilar ordering of theories phrased in a propositional language, British J. Philos. Sci. 38, 533-549.

M. Fitting [1989], Bilattices and the theory of truth, J. Philos. Logic 18, 225-256.

J. Fox [1990], Motivation and demotivation of a four-valued logic, Notre Dame J. Formal Logic $31(1), 76-80$.

H. Levesque [1984], A logic of implicit and explicit belief, in: Proceedings AAAI-84, Austin, TX, 198-202.

R. K. Meyer and E. Martin [1986], Logic on the Australian plan, J. Philos. Logic 15, 305-332.

P. F. Patel-Schneider [1989], A four-valued semantics for terminological logics, Artificial Intelligence 38, 319-351.

- [199?], A decidable first-order logic for knowledge representation, manuscript, AI Principles Research Department, AT\&T Bell Laboratories (October 1989). (Revised version from Proc. 9th Internat. Joint Conf. on AI (Los Angeles, California, 1985).) To appear in J. Automat. Reason.

H. Rasiowa [1974], An algebraic approach to non-classical logics, Stud. Logic Found. Math. 78, North-Holland, Amsterdam.

R. Sylvan [199?], On interpreting truth tables, and relevant truth table logic, to appear in Notre Dame J. Formal Logic. 